\title{
Miniature Dual-Band Substrate Integrated Waveguide Slotted Antenna Array for Millimeter-Wave 5G Applications
}

\author{
Fei-Peng Lai, Lu-Wu Chang, and Yen-Sheng Chen \\ Department of Electronic Engineering, National Taipei University of Technology, Taipei 10608, Taiwan \\ Correspondence should be addressed to Yen-Sheng Chen; yschen@ntut.edu.tw
}

Received 28 July 2020; Revised 8 September 2020; Accepted 30 September 2020; Published 12 October 2020

Academic Editor: Diego Caratelli

Copyright $\odot 2020$ Fei-Peng Lai et al. This is an open access article distributed under the Creative Commons Attribution License, which permits unrestricted use, distribution, and reproduction in any medium, provided the original work is properly cited.

\begin{abstract}
A compact substrate integrated waveguide (SIW) antenna array that operates at $28 \mathrm{GHz}$ and $38 \mathrm{GHz}$ is proposed for fifth generation $(5 \mathrm{G})$ applications. The proposed array consists of four SIW cavities fabricated on one single layer of substrate. Each cavity implements a rhombic slot and a triangular-split-ring slot, resonating on $\mathrm{TE}_{101}$ and $\mathrm{TE}_{102}$ modes at $28 \mathrm{GHz}$ and $38 \mathrm{GHz}$, respectively. In comparison with dual-band SIW antennas in the literature, the proposed configuration depicts a miniature footprint $\left(28.7 \times 30.8 \mathrm{~mm}^{2}\right)$ without stacking substrates. To excite the four cavities with equal power, a broadband power divider that supports the propagation of $\mathrm{TE}_{10}$ mode is designed. Accordingly, the impedance bandwidths are $26.6-28.3 \mathrm{GHz}$ and $36.8-38.9 \mathrm{GHz}$. The measured realized peak gain over the lower and higher bands is $9.3-10.9 \mathrm{dBi}$ and $8.7-12.1 \mathrm{dBi}$, respectively. The measured half-power beam widths (HPBWs) at $28 \mathrm{GHz}$ and $38 \mathrm{GHz}$ are $20.7^{\circ}$ and $15.0^{\circ}$, respectively. Considering these characteristics, including dual bands, high gain, narrow beam widths, miniaturization, and single layer, the proposed antenna array is a suitable candidate for millimeter-wave $5 \mathrm{G}$ communication systems with the flexibility in switching operating frequency bands against channel quality variations.
\end{abstract}

\section{Introduction}

The millimeter-wave fifth generation (5G) communications have gathered great importance in recent years. As relatively broad bandwidths are available at $28 \mathrm{GHz}$ and $38 \mathrm{GHz}$, millimeter-wave communications provide higher data capacity. To feed a millimeter-wave antenna, waveguide structures are particularly suitable due to low loss and high power handling capability. However, classical waveguides with solid walls have difficulty to be integrated with printed circuit boards (PCBs). In this situation, substrate integrated waveguide (SIW) feeds can be fabricated with PCBs seamlessly, showing the advantages of low material and fabrication cost, low profile, and excellent shielding properties. Thus, SIW antennas depict great potential for $5 \mathrm{G}$ millimeter-wave applications.

Although considerable attention has been paid to the development of 5G millimeter-wave SIW antennas, earlier studies put more emphasis on single-band design at $28 \mathrm{GHz}$ [1-10]. Simultaneous realization of $28-\mathrm{GHz}$ and $38-\mathrm{GHz}$ resonances can reduce the cost and space, but such dualband designs are relatively lacking [11-14]. The performance of these dual-band SIW antenna arrays is shown in Table 1. Several arrays are implemented through multiple layers of substrates [11-13], which increase the complexity and cost of integration. On the other hand, the single-layer design depicts relatively large dimensions, and the impedance bandwidths are too narrow to cover the $5 \mathrm{G}$ specification [14].

The goal of this paper is to present a miniature dual-band SIW slotted array fabricated on one single layer of substrate for millimeter-wave 5G applications. This SIW antenna consists of four cavities arranged as a linear array. The novelty of the proposed antenna array is threefold. First, while SIW slotted arrays have been constructed by conventional thin slots $[4,6-8,12,14]$, square rings $[3,5]$, quarter-mode resonant cavities [13], triangular-split-ring slots [10], and spoon-shaped slots [9], this study proposes a cavity that consists of new resonant topologies, including a rhombic slot and a triangular-split-ring slot. This new 
TABle 1: Comparison of dual-band SIW antenna arrays $(28 / 38 \mathrm{GHz})$.

\begin{tabular}{lcccc}
\hline No. & Number of cavities & Antenna footprint $\left(\mathrm{mm}^{2}\right)$ & Multiple layers? & Impedance bandwidths $(\mathrm{GHz})$ \\
\hline$[11]$ & $2 \times 2$ & $105.0 \times 83.7$ & Yes & $24.2-27.8,36.9-42.8$ \\
{$[12]$} & $2 \times 2$ & $32.0 \times 40.2$ & Yes & $26.3-32.3,37.4-38.4$ \\
{$[13]$} & $1 \times 4$ & $27.5 \times 28.5$ & Yes & $26.7-30.4,36.6-38.8$ \\
{$[14]$} & $1 \times 4$ & $40.0 \times 27.5$ & No & $28.0-28.3,37.3-39.1$ \\
This study & $1 \times 4$ & $28.7 \times 30.8$ & No & $26.6-28.3,36.8-38.9$ \\
\hline
\end{tabular}

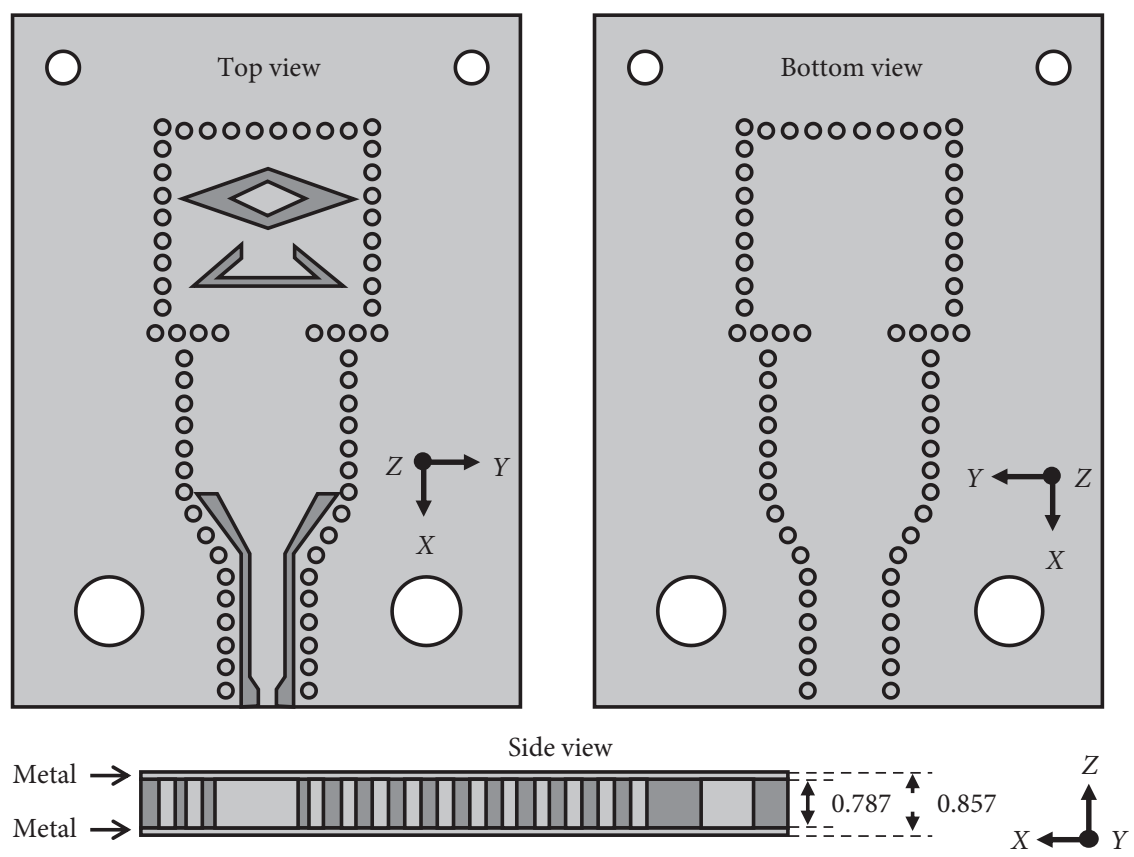

(a)

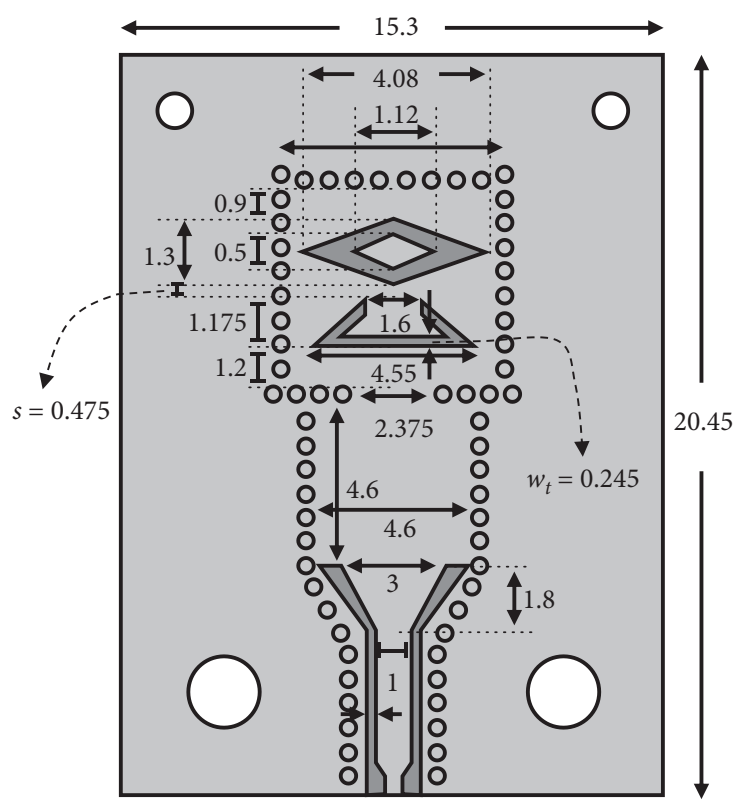

(b)

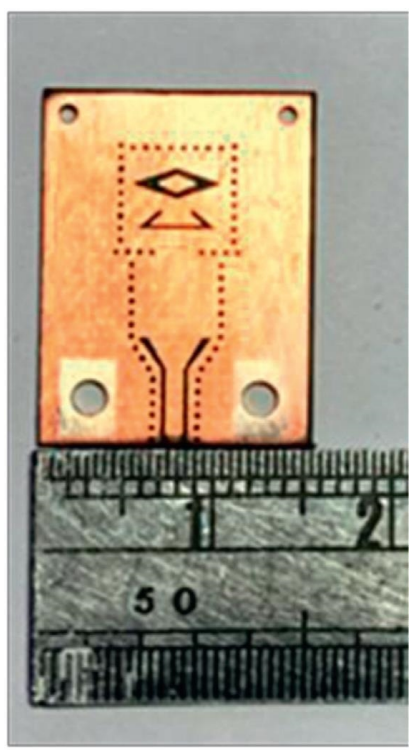

(c)

Figure 1: (a) Geometry, (b) detailed dimensions, and (c) photograph of the proposed unit element (unit: mm).

topology miniaturizes the antenna footprint, providing straightforward integration with the microwave electronics of a transceiver. Next, the proposed antenna is a single-layer design, reducing cost and complexity from multilayer PCBs [11-13]. Third, although a miniature and low-profile antenna is developed, the antenna still provides sufficient 
impedance bandwidths and high broadside gain. The operating mechanism of the antenna will be explained, and optimized performances supplemented with simulated and measured results will be demonstrated.

\section{Unit Element}

While the proposed antenna is a $1 \times 4$ linear array, this section presents the design and analysis of a unit element. Figure 1 exhibits the geometry and detailed dimensions of the proposed unit element. The antenna is fabricated on one layer of RT/Duroid 5880 substrate (dielectric constant $\varepsilon_{\mathrm{r}}=2.2$ and loss tangent $\tan \delta=0.003$ ). The SIW is fabricated by metallic via arrays, where the separation between adjacent vias is $0.675 \mathrm{~mm}$ and the diameter is $0.4 \mathrm{~mm}$. A tapered SIWto-grounded co-planar waveguide (GCPW) transition is designed to enable dual-band features. The GCPW is terminated with a $50 \Omega$ end launcher, which is utilized to excite the cavity.

The proposed unit element consists of a SIW cavity with a rhombic slot and a triangular-split-ring slot. To explain the principle of dual-band resonances, Figure 2 exhibits the distributions of E-fields simulated using Ansoft HFSS. With the SIW-to-GCPW transition, the TEM mode in the GCPW can be converted into $\mathrm{TE}_{10}$ fundamental mode in the SIW. More explicitly, Figure 3 demonstrates the E-field distributions of the cavity at $28 \mathrm{GHz}$ and $38 \mathrm{GHz}$. The maximum magnitude at $28 \mathrm{GHz}$ is associated with both the resonators. The E-field of the rhombic slot is closely coupled with that of the triangularsplit-ring slot, leading to mode bifurcation at the resonant frequency. The cavity resonates on $\mathrm{TE}_{101}$ mode. At $38 \mathrm{GHz}$, the maximum magnitude is majorly radiated through the triangular-split-ring slot, and the cavity resonates on $\mathrm{TE}_{102}$ mode.

The operating principle explained above can be validated through parametric studies. Figure 4 indicates that the impedance characteristics at $28 \mathrm{GHz}$ and $38 \mathrm{GHz}$ are affected by the variation of the width of the triangularsplit-ring slot, namely, $w_{t}$. The larger the $w_{t}$, the higher the resonant frequencies are for both the dual bands. In addition, when the separation between the two slots, namely, $s$, is varied over $0.425 \mathrm{~mm}, 0.475 \mathrm{~mm}$, and $0.525 \mathrm{~mm}$, the impedance matching at $28 \mathrm{GHz}$ is unaffected. Meanwhile, the resonant frequency at the higher frequency band can be controlled by tuning $s$; the associated resonant frequencies are $38.8 \mathrm{GHz}, 38.6 \mathrm{GHz}$, and $38.2 \mathrm{GHz}$, respectively. According to these results, the design rule of the proposed unit element involves two steps. The first step is to tune the geometric parameters to cover the lower frequency band. When the bandwidth requirement of the lower band is met, the second step is to tune the geometric parameters of the triangular-split-ring slot so that the bandwidth of the higher frequency band covers the specification and that of the lower band remains unvaried. These design guidelines offer us a systematic method to obtain the configuration of the rhombic slot and the triangular-split-ring slot. In addition, our parametric studies suggest that the maximum

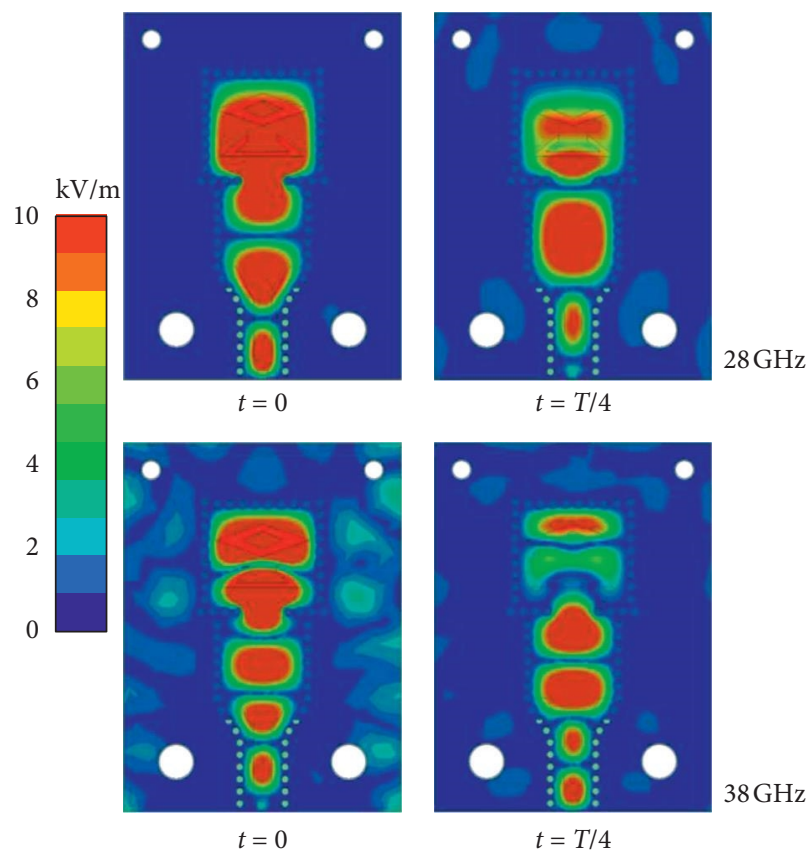

Figure 2: Distributions of the E-field of the proposed unit element.

dimension of the triangular-split-ring slot should be larger than $3.2 \mathrm{~mm}$ to cover the lower frequency band. Similarly, the maximum dimension of the rhombic slot should be larger than $4.3 \mathrm{~mm}$ to cover the higher frequency band.

The prototype of the proposed unit element is fabricated and tested. The impedance characteristics are tested using a vector network analyzer (VNA) N52227A from Keysight. Figure 5 shows the simulated and measured reflection coefficients. In terms of the $-10 \mathrm{~dB}$ threshold, the simulated impedance bandwidths are $27.7-28.4 \mathrm{GHz}$ and $37.7-40.1 \mathrm{GHz}$, whereas the measured ones are $26.7-27.7 \mathrm{GHz}$ and $36.7-38.2 \mathrm{GHz}$. There are frequency shifts between the simulated and measured results. The discrepancy could be due to the manufacturing errors including the variation of the substrate thickness and the soldering of the end launcher. As the thickness of the substrate increases, the resonant frequency shifts to a lower level. The measured response agrees very well with the antenna designed with a $0.82 \mathrm{~mm}$-height substrate. On the other hand, the model in full-wave simulation does not include the end launcher, which causes differences between the simulated and measured results. The sensitive nature of millimeter-wave cavity and resonator geometry also incurs additional errors.

Figure 6 exhibits the simulated and measured realized peak gain and antenna efficiency. For the lower frequency band, the simulated (measured) realized peak gain is 7.6-8.5 (7.0-8.7) $\mathrm{dBi}$. At the resonant frequency, the simulated and measured antenna efficiencies are $84 \%$ and $82 \%$, respectively. For the higher frequency band, the simulated (measured) realized peak gain varies over 7.0-8.0 (3.9-6.8) $\mathrm{dBi}$. At the resonant frequency, the simulated and measured antenna efficiencies are $76 \%$ and 


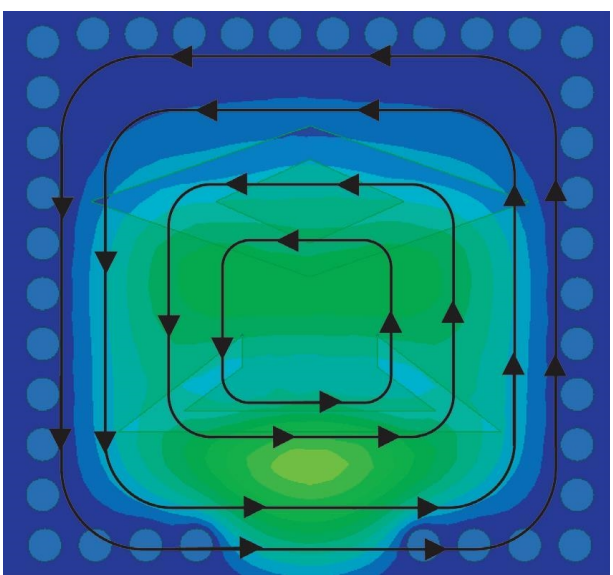

(a)

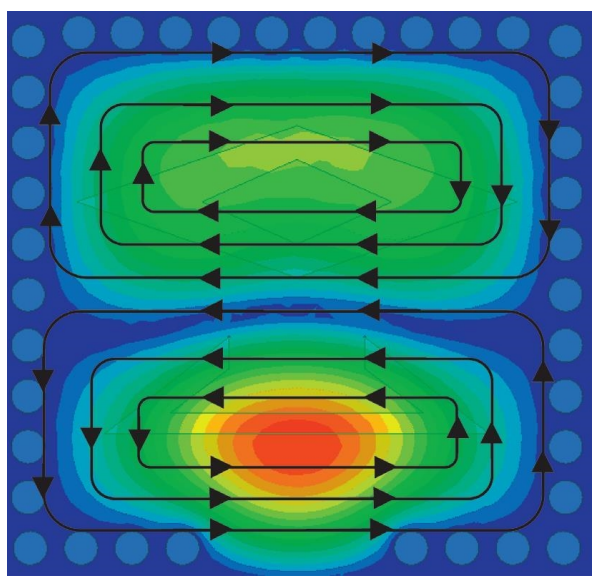

(b)

Figure 3: Distributions of the E-field inside the cavity: (a) $\mathrm{TE}_{101}$ at $28 \mathrm{GHz}$ and (b) $\mathrm{TE}_{102}$ at $38 \mathrm{GHz}$.

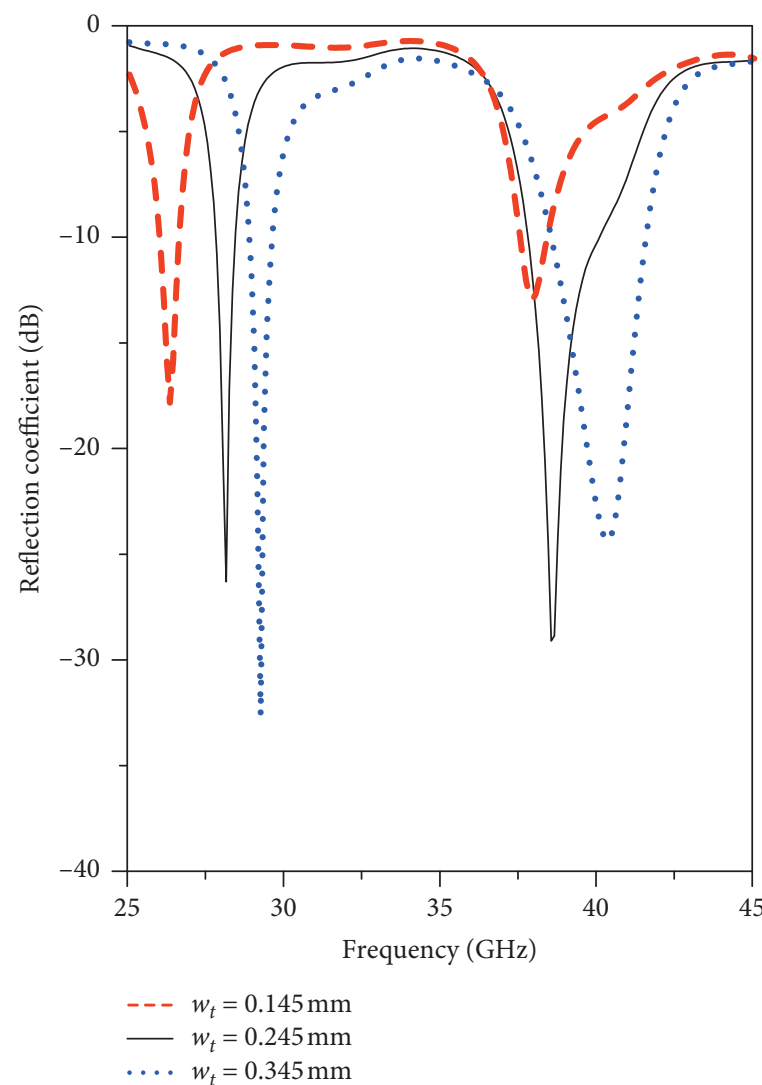

(a)

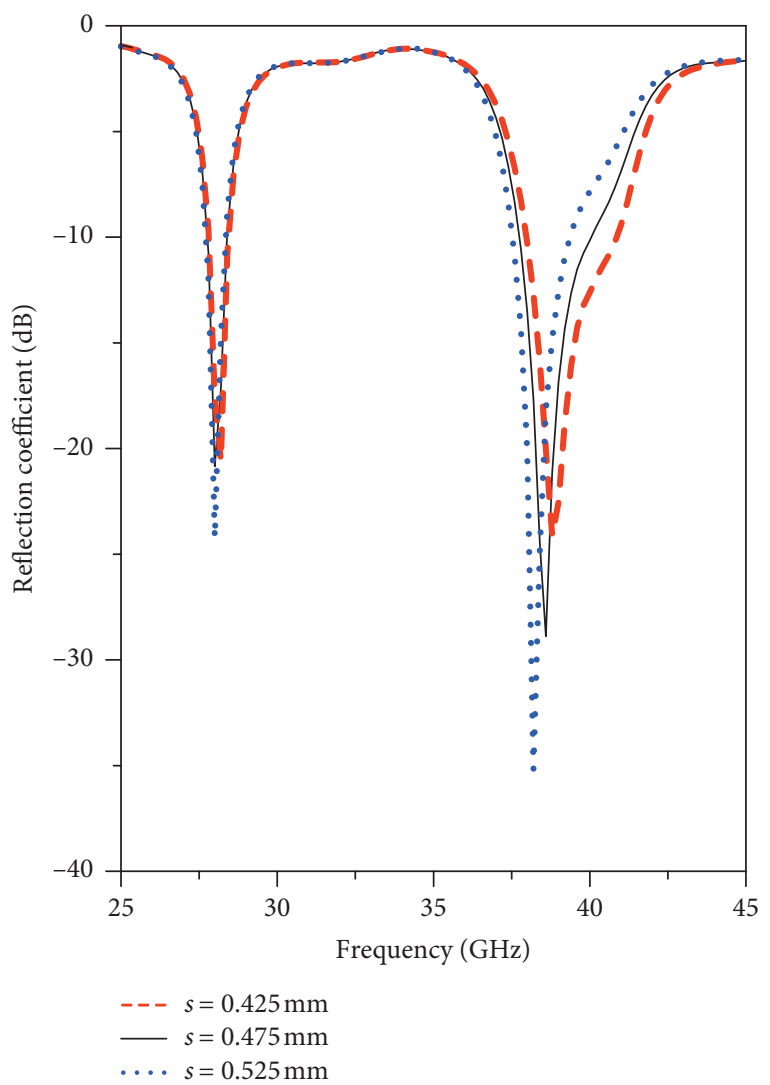

(b)

Figure 4: Parametric analysis of the proposed unit element. Effect of the variation of (a) $w_{t}$ and (b) $s$.

$37 \%$, respectively. The far-field experiments are performed in an anechoic chamber based on compact antenna test range (CATR), as shown in Figure 7 . The discrepancy between the simulated efficiency and the measured one is noticeable at the $38 \mathrm{GHz}$ band. This difference is attributed to three issues. First, as will be shown in Figure 8, the pattern of the unit element is not highly directional, and the half-power beam widths (HPBWs) are greater than $70^{\circ}$. Thus, mutual coupling between the AUT and the transmitting horn antenna may occur, reducing the radiation efficiency of the AUT. Second, serrated structures are not implemented in the available CATR anechoic chamber, so diffraction effects may reduce the measurement resolution and the antenna 


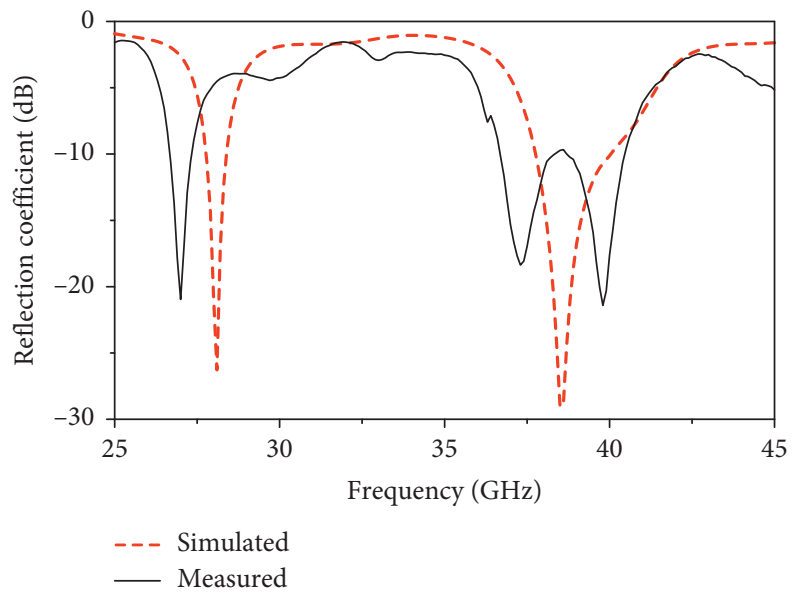

FigurE 5: Simulated and measured reflection coefficients of the proposed unit element.

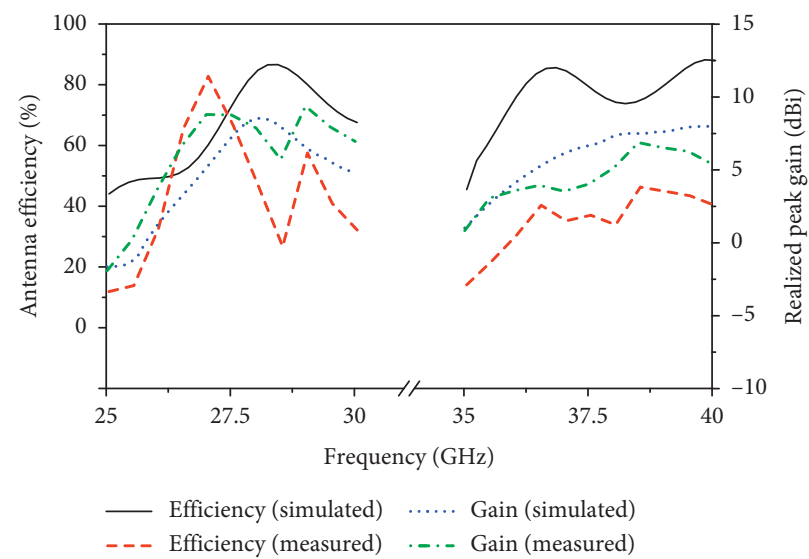

Figure 6: Antenna efficiency and realized peak gain of the proposed unit element.

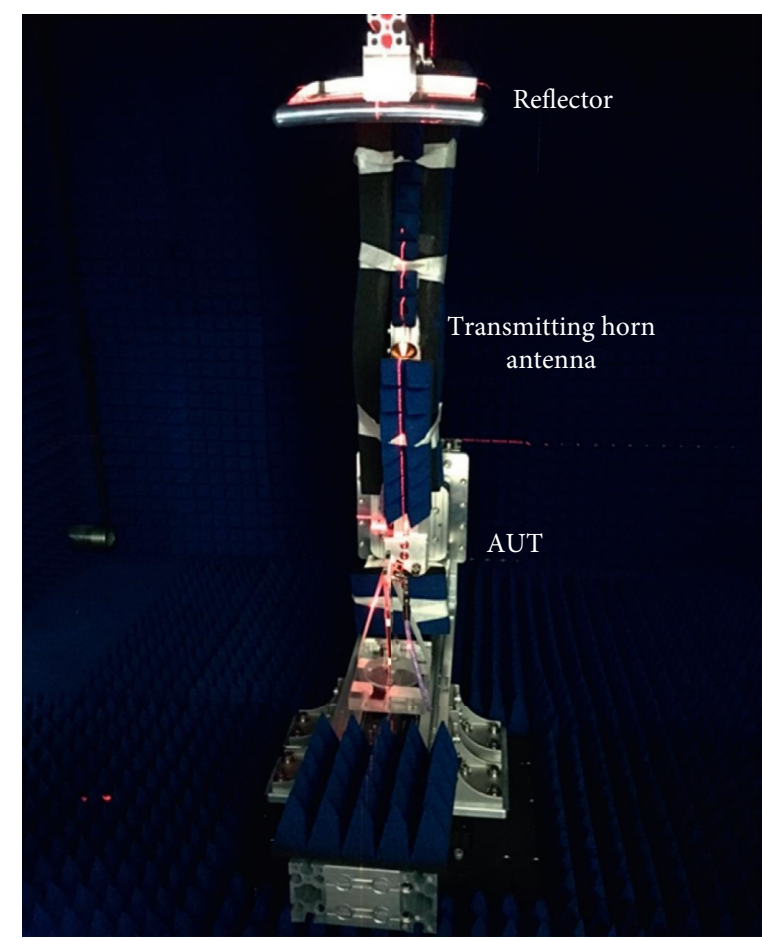

FigURe 7: Far-field measurement setup in a compact-range anechoic chamber. 

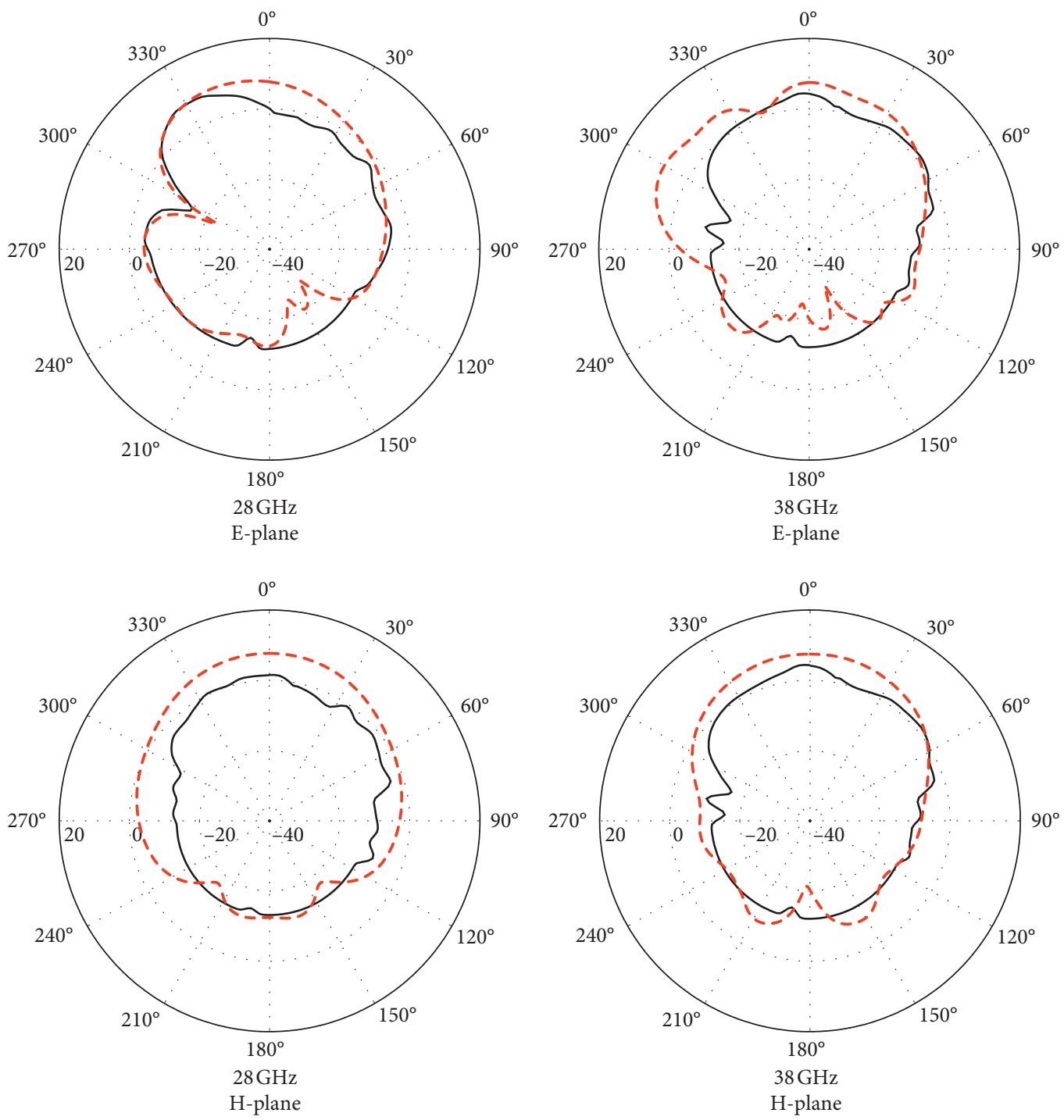

- - - Simulated

- Measured

FIGURE 8: Simulated and measured radiation patterns of the proposed unit element (unit: $d B i$ ).

efficiency. Third, the simulated efficiency does not include the loss due to the end launcher, but the imperfect conductivity of the connector may incur additional loss, especially at $38 \mathrm{GHz}$.

More explicitly, Figure 8 demonstrates the simulated and measured radiation patterns. The main beam is directed at the broadside direction. The simulated (measured) HPBWs at the resonant frequencies of the lower and higher bands are $70.5^{\circ}\left(76.0^{\circ}\right)$ and $76.2^{\circ}\left(74.7^{\circ}\right)$, respectively. These impedance and radiation characteristics validate the proposed unit element.

\section{Antenna Array}

After investigating the performance of the unit element, four identical unit elements are integrated as a linear array. Figure 9 exhibits the geometry and detailed dimensions of the proposed $1^{\circ} \times^{\circ} 4$ slotted SIW antenna array. The four cavities are cascaded in a shunt-fed manner. All design parameters regarding a single cavity are the same as those described in the previous section.

A broadband power divider is designed to excite the four cavities. The design is created by the duplicate of multiple 2-way dividers. Considering a 2-way power divider, tailoring the vertical segments leads to the equality of output power at each port, and adjusting the spacing between horizontal segments results in an antiphase between adjacent ports. The E-field distributions of the power divider are shown in Figure 10. As compared to the E-field of the unit element, the proposed slotted array still can be excited by the $\mathrm{TE}_{10}$ mode. In addition, this power divider avoids multilevel divider configurations, splitting input energy into 4-way output straightforwardly.

For verification, the proposed SIW slotted antenna array is tested in terms of impedance and radiation characteristics. 


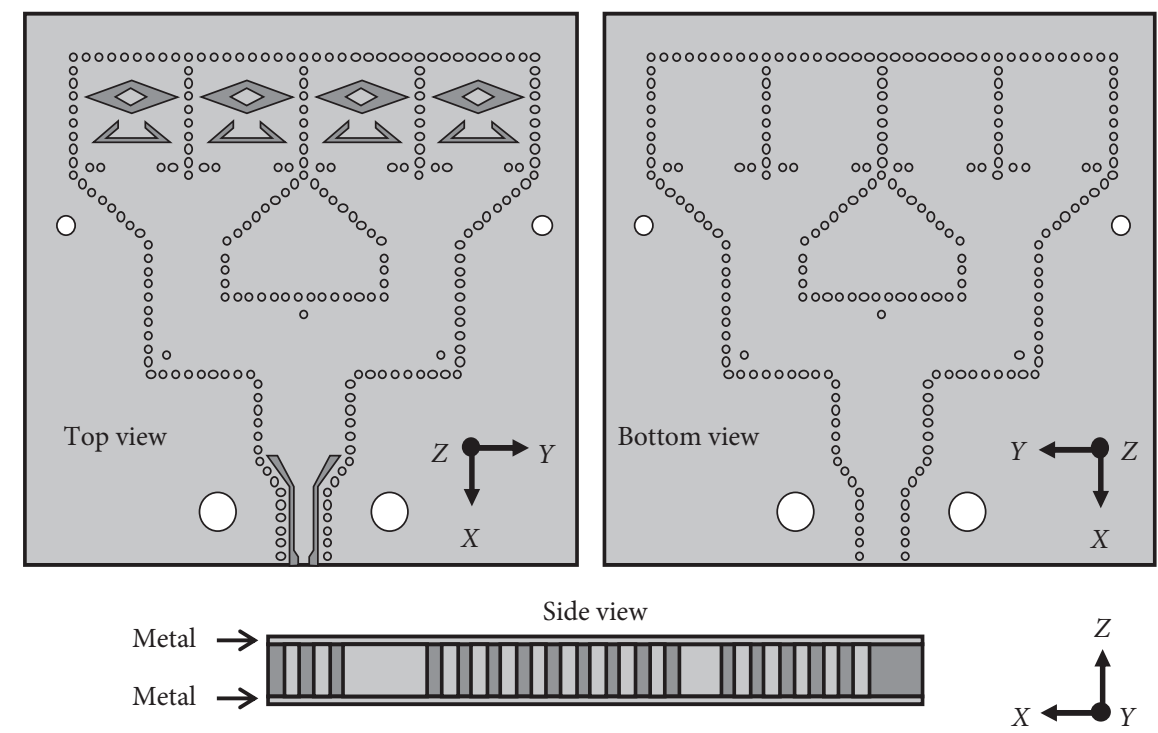

(a)

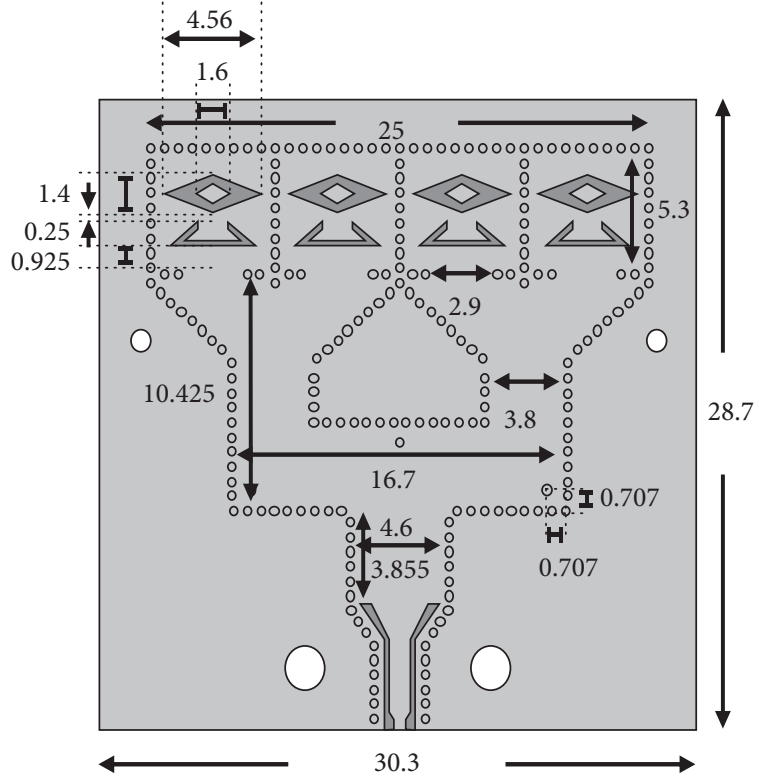

(b)

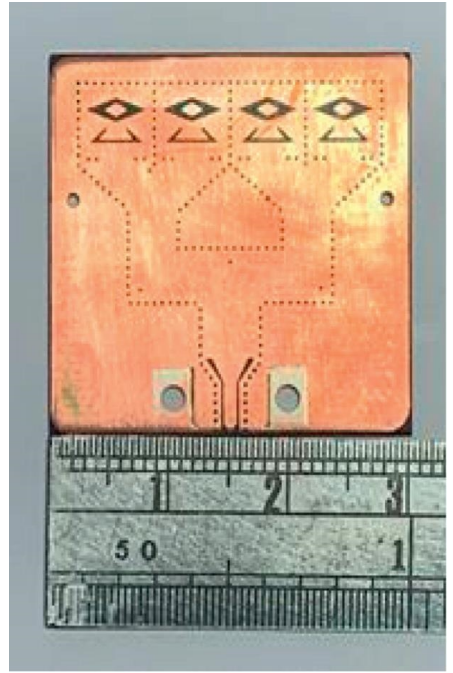

(c)

Figure 9: (a) Geometry, (b) detailed dimensions, and (c) photograph of the proposed SIW slotted antenna array (unit: mm).

The simulated (measured) reflection coefficients, provided in Figure 11, indicate that the impedance bandwidths are $26.6-28.4 \mathrm{GHz}$ and $37.8-39.3 \mathrm{GHz}(26.6-28.3 \mathrm{GHz}$ and $36.8-38.9 \mathrm{GHz}$ ). Minor discrepancies are observed, and they are attributed to the experimental manufacturing techniques. However, both the millimeter-wave 5G frequency bands can be covered by the proposed antenna array.

Figure 12 depicts the realized peak gain and antenna efficiency. The simulated peak gain and measured peak gain over the lower frequency band are $8.5-11.8 \mathrm{dBi}$ and 9.3-10.9 $\mathrm{dBi}$, respectively, whereas the simulated and measured results over the higher frequency band are $13.5-14.3 \mathrm{dBi}$ and $8.7-12.1 \mathrm{dBi}$. The simulated (measured) antenna efficiency at the resonant frequencies of the lower and higher bands is 66\% (45\%) and 72\% (41\%), respectively.

Figure 13 exhibits radiation patterns at $28 \mathrm{GHz}$ and $38 \mathrm{GHz}$. The broadside gain at $28 \mathrm{GHz}$ and $38 \mathrm{GHz}$ is $9.2 \mathrm{dBi}$ and $8.7 \mathrm{dBi}$, respectively. In particular, at $28 \mathrm{GHz}$, the simulated and measured HPBWs on the H-plane are $20.4^{\circ}$ and $20.7^{\circ}$, respectively. At $38 \mathrm{GHz}$, the simulated and measured HPBWs on the H-plane are $15.3^{\circ}$ and $15.0^{\circ}$, respectively. Additional studies are performed to investigate the radiation performance of the proposed $1 \times 4$ layout and that of a $2 \times 2$ series-fed SIW slotted array. The proposed design demonstrates desirable broadside radiation, whereas the $2 \times 2$ series-fed layout suffers from the deflation angle of main beams. Since dual-band performance is pursued, 


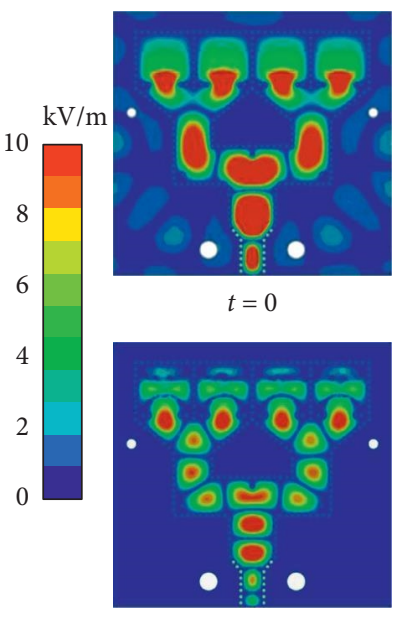

$t=0$

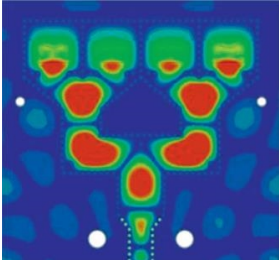

$28 \mathrm{GHz}$

$t=T / 4$

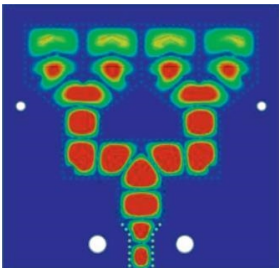

$t=T / 4$

$38 \mathrm{GHz}$

FiguRE 10: Distributions of the E-field of the proposed antenna array.

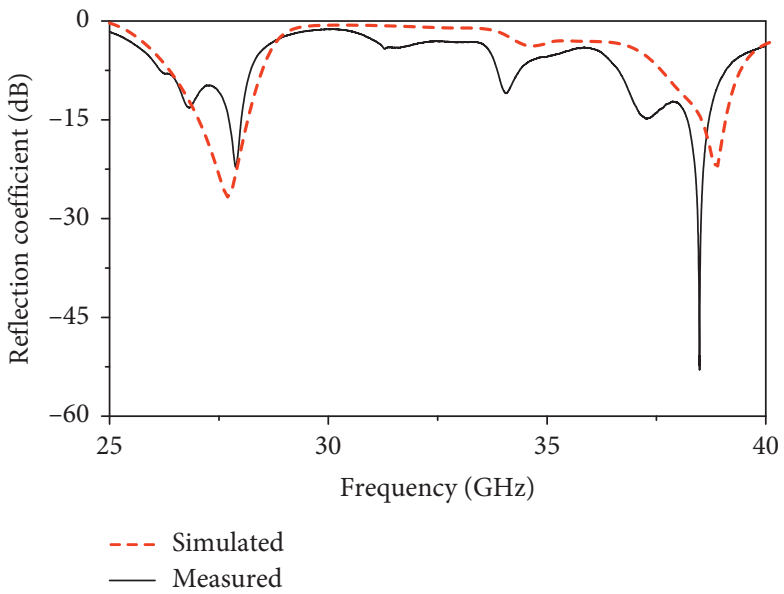

FIGURE 11: Simulated and measured reflection coefficients of the proposed antenna array.

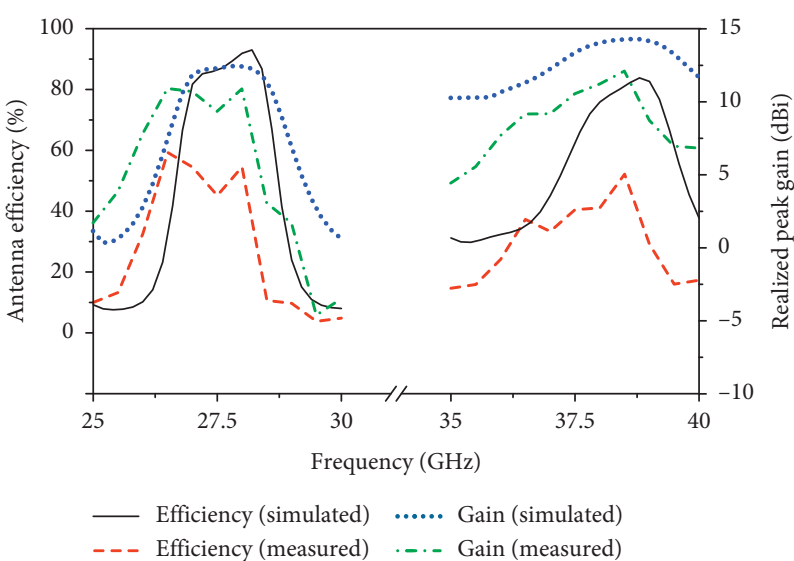

Figure 12: Antenna efficiency and realized peak gain of the proposed antenna array. 

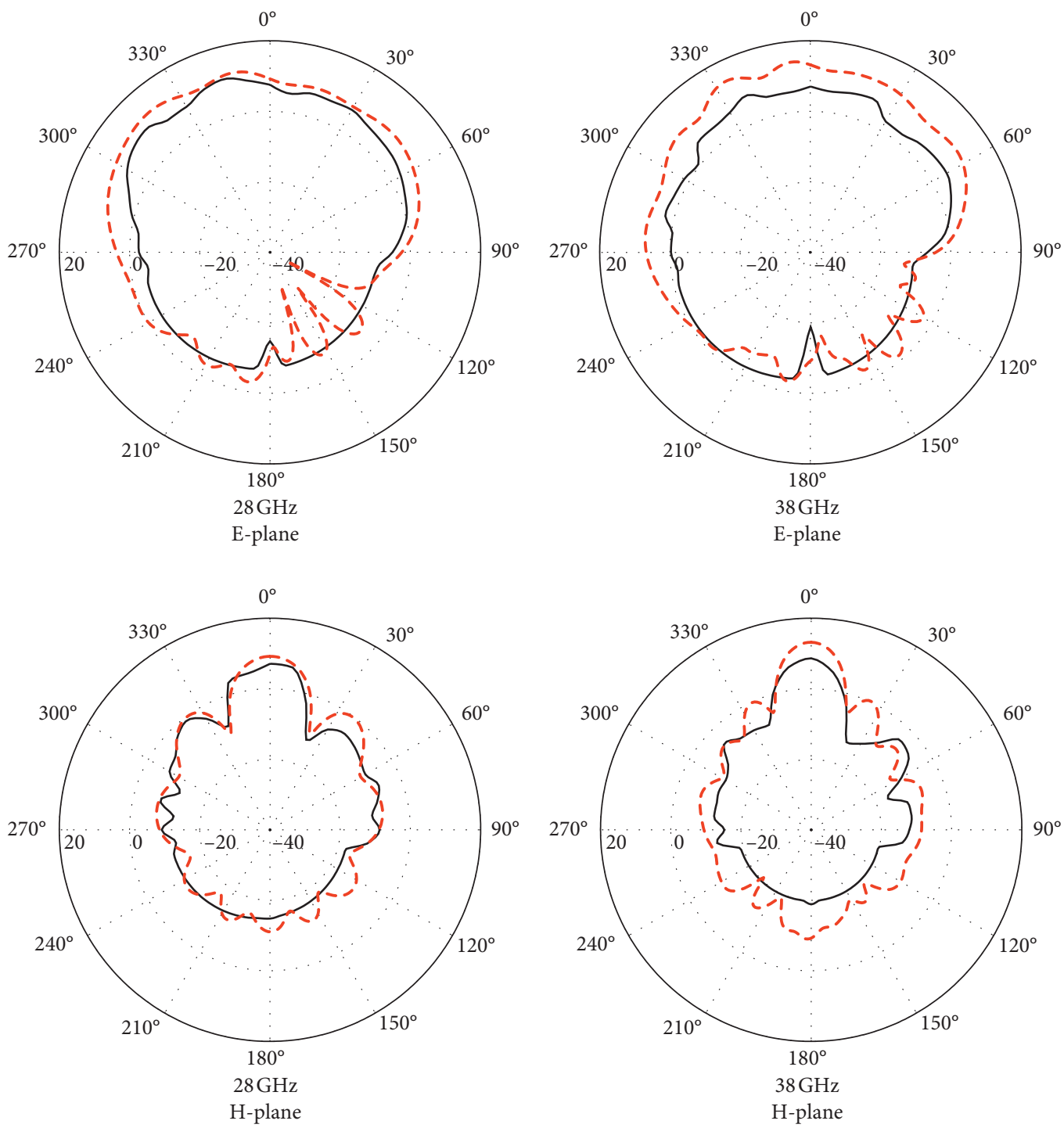

- - - Simulated

- Measured

FIGURE 13: Simulated and measured radiation patterns of the proposed antenna array (unit: dBi).

creating in-phase excitations for longitudinal adjacent elements on both the frequency bands is challenging. These results indicate that the proposed antenna achieves dualband, high-gain, and narrow-beam features.

\section{Conclusion}

A dual-band SIW slotted antenna array that implements four cavities comprised of rhombic and triangular-split-ring slots has been presented. The proposed topology has three distinct features. First, the $1 \times 4$ antenna array demonstrates a compact footprint $\left(28.7 \times 30.8 \mathrm{~mm}^{2}\right)$ with single-layer construction, reducing manufacturing complexity and providing straightforward integration. Second, a systematic design method is developed for the proposed antenna. The resonance at the lower frequency band is generated by the mode bifurcation of the rhombic and triangular-split-ring slots, whereas the higher resonant frequency can be controlled by tuning the triangular-split-ring slot alone. Third, the proposed design not only shows manufacturing simplicity, but it also provides sufficient impedance bandwidths $(26.6-28.3 \mathrm{GHz}$ and $36.8-38.9 \mathrm{GHz})$ and high realized gain $(10.9 \mathrm{dBi}$ and $12.1 \mathrm{dBi}$ for the lower and higher frequency bands, respectively). These results make the proposed SIW slotted antenna array a suitable candidate for $5 \mathrm{G}$ dual-band communication systems.

\section{Data Availability}

The simulated and measured data used to support the findings of this study are included within the article.

\section{Conflicts of Interest}

The authors declare that there are no conflicts of interest regarding the publication of this paper. 


\section{Acknowledgments}

This study was supported in part by the National Taipei University of Technology, Taiwan, under contract NTUTSZU-109-08 and in part by the Ministry of Science and Technology, Taiwan, under contract MOST 109-2636-E-027004 .

\section{References}

[1] K. S. Sultan, H. H. Abdullah, E. A. Abdallah, and H. S. ElHennawy, "Metasurface-based dual polarized MIMO antenna for 5G smartphones using CMA," IEEE Access, vol. 8, pp. 37250-37264, 2020.

[2] N. Al-Fadhali, H. A. Majid, R. Omar, M. K. A. Rahim, S. M. Shah, and B. A. F. Esmail, "Wideband (22-30 GHz) Mmwave substrate integrated waveguide (SIW) antenna for $5 \mathrm{G}$ applications," in Proceedings of the IEEE Conference on Antenna Measurements \& Applications (CAMA), pp. 1-4, Kuta, Indonesia, October 2019.

[3] R. Parthasarathy, A. Chandrasekar, and P. G. V Ramesh, "Design of linear $2 \times 2$ array using substrate-integratedwaveguide patch antenna for $28 \mathrm{GHz} \mathrm{Mm}$-wave applications," in Proceedings of the 2019 TEQIP III Sponsored International Conference on Microwave Integrated Circuits, Photonics and Wireless Networks (IMICPW), pp. 44-49, Tiruchirappalli, India, May 2019.

[4] R. B. Di Renna, V. P. R. Magri Souza, T. N. Ferreira, L. J. Matos, J. A. M. Souza, and G. L. Siqueira, "A new doublesided substrate-integrated waveguide slot array antenna for 5G applications," Microwave and Optical Technology Letters, vol. 61, no. 3, pp. 682-687, 2019.

[5] M. Shirichian, G. Moradi, R. S. Shirazi, and V. Hassanzade, "Design a circularly polarized SIW slot antenna for 5G base stations," in Proceedings of the 2018 Fifth International Conference on Millimeter-Wave and Terahertz Technologies (MMWaTT), pp. 82-85, Tehran, Iran, December 2018.

[6] P. Jing, B. Li, B. Wu, N. Zhang, Y. Xu, and X. Lv, "A millimeterwave SIW slot antenna array for 5G communication," in Proceedings of the 2018 International Conference on Microwave and Millimeter Wave Technology (ICMMT), pp. 1-3, Chengdu, China,, May 2018.

[7] S. Ho-Quang, S. X. Ta, P. Huynh-Nguyen-Bao, K. NguyenKhac, and C. Dao-Ngoc, "Compact circularly polarized slotted SIW cavity antenna for 5G application," in Proceedings of the 2017 International Conference on Advanced Technologies for Communications (ATC), pp. 75-79, Quy Nhon, Vietnam, October 2017.

[8] M. Asaadi and A. Sebak, "High-gain low-profile circularly polarized slotted SIW cavity antenna for MMW applications," IEEE Antennas and Wireless Propagation Letters, vol. 16, pp. 752-755, 2016.

[9] Q. Wu, H. Wang, C. Yu, and W. Hong, "Low-profile circularly polarized cavity-backed antennas using SIW techniques," IEEE Transactions on Antennas and Propagation, vol. 64, no. 7, pp. 2832-2839, 2016.

[10] P. N. Choubey, W. Hong, Z.-C. Hao, P. Chen, T.-V. Duong, and J. Mei, "A wideband dual-mode SIW cavity-backed triangular-complimentary-split-ring-slot (TCSRS) antenna," IEEE Transactions on Antennas and Propagation, vol. 64, no. 6, pp. 2541-2545, 2016.

[11] B. Feng, X. He, J.-C. Cheng, and C.-Y.-D. Sim, "Dual-wideband dual-polarized metasurface antenna array for the $5 \mathrm{G}$ millimeter wave communications based on characteristic mode theory," IEEE Access, vol. 8, pp. 21589-21601, 2020.

[12] T. Hong, Z. Zhao, W. Jiang, S. Xia, Y. Liu, and S. Gong, "Dualband SIW cavity-backed slot array using TM020 and TM120 modes for 5G applications," IEEE Transactions on Antennas and Propagation, vol. 67, no. 5, pp. 3490-3495, 2019.

[13] T. Deckmyn, M. Cauwe, D. Vande Ginste, H. Rogier, and S. Agneessens, "Dual-band $(28,38) \mathrm{GHz}$ coupled quartermode substrate-integrated waveguide antenna array for nextgeneration wireless systems," IEEE Transactions on Antennas and Propagation, vol. 67, no. 4, pp. 2405-2412, 2019.

[14] N. Ashraf, O. Haraz, M. A. Ashraf, and S. Alshebeili, "28/38$\mathrm{GHz}$ dualband millimeter wave SIW array antenna with EBG structures for 5G applications," in Proceedings of the Information and Communication Technology Research (ICTRC), pp. 5-8, Abu Dhabi, United Arab Emirates, May 2015. 